\title{
Tendencias Clínico-Hospitalarias de Usuarios en Servicios de Salud Mental
}

\section{Clinical-Hospital Trends in Users of Mental Health Services Tendências Clínico-Hospitalares dos Usuários em Serviços de Saúde Mental}

doi http://dx.doi.org/10.35954/SM2019.38.2.2

\author{
María Fernanda Olivera a (D) https://orcid.org/0000-0003-0092-0942

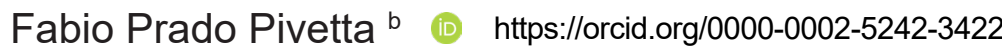

(a) Servicio de Traumatología Quirúrgica, Hospital Central de las Fuerzas Armadas.

(b) Departamento de Salud Mental, Psiquiatría, Hospital Central de las Fuerzas Armadas.

\section{RESUMEN}

El Hospital Central de las Fuerzas Armadas de la República Oriental del Uruguay en su servicio de internación psiquiátrica, asiste a su personal en actividad, retirados y familiares directos. La ausencia de investigaciones propias (datos procesados) del servicio ha sido piedra angular en la realización de este trabajo. Con el propósito de generar una metodología y poder comparar tendencias a nivel regional es que se planteó el objetivo de encontrar similitudes o diferencias con el servicio de psiquiatría de la Clínica Universidad Católica de San Carlos de Apoquindo de Santiago de Chile. Describimos y conocimos las características demográficas, los principales diagnósticos de ingreso, la frecuencia de readmisión y se promedió los días de internación desde la base de datos de las hojas de admisión, sistema informático del Departamento de Estadística y Registros Médicos y el cuaderno de registro de ingresos y egresos del servicio. La información fue procesada en planilla de Microsoft Excel 97-2003 con un total de 192 registros en el período de un año. Las patologías más frecuentes fueron dentro de los grupos de Esquizofrenias y Trastornos Delirantes, Trastornos del Humor (Afectivos); Trastornos de Personalidad con Trastornos del Comportamiento y en cuarto lugar el Trastorno Mental y del Comportamiento debido al Consumo de Sustancias. La readmisión se da sobre todo en usuarios con patologías crónicas recurrentes dentro de los sub grupos trastornos delirantes y del humor.

Palabras Clave: Admisión del Paciente; Hospitalización; Readmisión del Paciente; Salud Mental; Trastornos Mentales.

\section{ABSTRACT}

The Central Hospital of the Armed Forces of the Eastern Republic of Uruguay, in its psychiatric internment service, assists its active personnel, retirees and direct relatives. The absence of the service's own investigations (processed data) has been a cornerstone in the performance of this work. In order to generate a methodology and be able to compare trends at a regional level, the objective was to find similarities or differences with the psychiatry service of the Catholic University Clinic of San Carlos de Apoquindo in Santiago de Chile. We described and learned the demographic characteristics, the main admission diagnoses, the frequency of readmission and the average days of hospitalization from the database of 
the admission sheets, the computer system of the Department of Statistics and Medical Records and the logbook of income and expenses of the service. The information was processed in Microsoft Excel spreadsheet 97-2003 with a total of 192 records in the period of one year. The most frequent pathologies were within the groups of Schizophrenia and Delusional Disorders, Humor Disorders (Affective); Personality Disorders with Behavioral Disorders and fourthly Mental Disorders and Behavioral Disorders due to Substance Use. Readmission occurs mainly in users with recurrent chronic pathologies within the subgroups delirious and mood disorders.

KEY WORDS: Patient Admission; Hospitalization; Patient Readmission; Mental Health; Mental Disorders.

\section{RESUMO}

O Hospital Central das Forças Armadas da República Oriental do Uruguai, em seu serviço de internação psiquiátrica, atende a seu pessoal ativo, aposentados e parentes diretos. A ausência de investigações próprias do serviço (dados tratados) tem sido uma pedra angular na realização deste trabalho. A fim de gerar uma metodologia e poder comparar tendências em nível regional, o objetivo foi encontrar semelhanças ou diferenças com o serviço de psiquiatria da Clínica da Universidade Católica de San Carlos de Apoquindo em Santiago do Chile. Descrevemos e aprendemos as características demográficas, os principais diagnósticos de internação, a frequência de readmissão e os dias médios de internação a partir do banco de dados das fichas de internação, do sistema informatizado do Departamento de Estatística e Registros Médicos e do diário de receitas e despesas do serviço. A informação foi processada na folha de cálculo Microsoft Excel 97-2003 com um total de 192 registos no período de um ano. As patologias mais frequentes foram dentro dos grupos de Esquizofrenia e Transtornos Delirantes, Transtornos de Humor (Afetivos); Transtornos de Personalidade com Transtornos Comportamentais e, em quarto lugar, Transtornos Mentais e Transtornos Comportamentais devido ao Uso de Substâncias. A readmissão ocorre principalmente em usuários com patologias crônicas recorrentes dentro dos subgrupos de delírios e transtornos de humor.

PAlavras Chave: Admissão do Paciente; Hospitalização; Readmissão do Paciente; Saúde Mental; Transtornos Mentais.

\section{INTRODUCCIÓN}

La salud mental y la comparación entre perfiles de usuarios internados en Psiquiatría, Sala 11, de la División Salud Mental del Hospital Central de las Fuerzas Armadas (H.C.FF.AA.) durante el período diciembre de 2017 a noviembre de 2018 con datos publicados en un centro chileno de similares características, es el análisis que presentaremos. Conocer la caracterización de usuarios internados, describiendo estadísticamente los criterios de internación según la Clasificación Estadística Internacional de Enfermedades y Problemas Relacionados con la Salud (CIE-10) $(1,2)$, se agrupa a la población en franjas etarias, sexo, días de hospitalización, readmisión de usuarios para realizar la comparación con otro servicio a nivel regional.

La salud mental se define como un estado de bienestar en el cual el individuo es consciente de sus propias capacidades, puede afrontar las tensiones normales de la vida y trabajar de forma productiva y fructífera siendo capaz de hacer una contribución a su comunidad. La dimensión positiva de la salud mental se destaca en la definición de salud que figura en la Constitución de la Organización Mundial de la Salud (OMS): "La salud es un estado de completo bienestar físico, mental y 
social, y no solamente la ausencia de afecciones o enfermedades" (3).

La salud mental incluye nuestro bienestar emocional, psicológico y social. Afecta la forma en que pensamos, sentimos y actuamos cuando enfrentamos la vida. También ayuda a determinar cómo manejamos el estrés, nos relacionamos con los demás y tomamos decisiones. Es importante en todas las etapas de la vida, desde la niñez y la adolescencia hasta la adultez. Las enfermedades mentales son afecciones graves que pueden alterar la manera de pensar, del humor y el comportamiento, pueden ser ocasionales o de larga duración, pueden afectar la capacidad de relacionarse con los demás y funcionar cada día. Los problemas mentales son comunes y existen tratamientos disponibles. Las personas con problemas de salud mental pueden mejorar y muchas de ellas se recuperan por completo $(3,4)$. Según Jaén Herrero la OMS habla de enfermedad como "Alteración o desviación del estado fisiológico en una o varias partes del cuerpo, por causas en general conocidas, manifestada por síntomas y signos característicos, y cuya evolución es más o menos previsible" (4).

La psiquiatría es la especialidad de la medicina que se encarga de la prevención, manejo y tratamiento de las enfermedades mentales en su totalidad. Sus contenidos nos obligan a un ejercicio de alta complejidad y está influenciado por el desarrollo de distintas áreas del conocimiento, desde las neurociencias hasta las ciencias sociales (5). Las enfermedades mentales se encuentran entre las más costosas debido a su alta prevalencia y cronicidad, temprana edad de aparición y discapacidad resultante. Desde los años 80 se han hecho propuestas de sistemas de clasificación que permiten ordenar los conocimientos y guiar la práctica clínica, pero aún hoy resultan muy insatisfactorios como el Manual diagnóstico y estadístico de los Trastornos Mentales (DSM-5) (6-9) y CIE-10 (1,2), debido a un cambio social en la interpretación de la salud donde se da lugar a la psiquiatrización de lo cotidiano, es decir, considerar patológicas, situaciones vitales como la tristeza, el miedo o el nerviosismo (10). Las Clasificaciones de las enfermedades son el fruto de un conocimiento científico limitado y de un consenso de investigadores y clínicos. La limitación tiene como consecuencia que hayan existido, y existen, clasificaciones hechas con criterios y desde perspectivas muy diferentes, unas veces etiológicas, otras evolutivas, otras psicopatológicas y otras, como el DSM-IV y la CIE-10 sintomáticas. Cada escuela y tradición de la psiquiatría tenía su clasificación o su hecho diferencial, como las psicosis psicógenas de la psiquiatría escandinava, las "bouffées delirantes" de la psiquiatría francesa o la esquizofrenia pseudoneurótica de la norteamericana, el consenso actual lo ha sustituido. Un consenso es un compromiso en aras de una aplicación práctica y de un lenguaje común. Suele ser el mejor de los mundos posibles o, si se quiere, de los mejores mundos, si tomamos en cuenta a la CIE-10 y el DMS-IV $(1,2,11)$.

El Proyecto de Salud Mental en su sigla en inglés MH "Mental Health" constituye una unidad técnica de la Organización Panamericana de la Salud (OPS), dentro del Departamento de Enfermedades no Transmisibles y Salud Mental en su sigla en inglés (NMH) "Noncommunicable Diseases and Mental Health", que promueve, coordina e implementa actividades de cooperación técnica dirigidas a fortalecer las capacidades nacionales para desarrollar políticas, planes, programas y servicios, contribuyendo a promover la salud mental, reducir la carga que significan las enfermedades psíquicas, prevenir las discapacidades y desarrollar la rehabilitación. Actualmente la mayoría o casi totalidad de los países de América Latina y el Caribe están recibiendo alguna modalidad de cooperación técnica en salud mental por parte de OPS/OMS, ya sea en proyectos específicos y de manera sistemática o de una forma puntual ante requerimientos concretos de los gobiernos (12). La rehospitalización de pacientes psiquiátricos hace referencia a la necesidad de internar nueva- 
mente a un paciente que ya había egresado por la misma causa. Es producto de una descompensación recurrente $y$, cuando se trata de patología mental crónica, las re hospitalizaciones obedecen a factores socio-demográficos, características clínicas y antecedentes personales. La tasa de rehospitalización permite hacer inferencias sobre la cantidad de individuos que no se reinsertan en forma adecuada a la comunidad y es un indicador de la calidad de la atención ambulatoria posterior al egreso. Indudablemente, el paciente sujeto a esta situación entra en un estado cíclico de reingresos que traen como consecuencia no poder participar en actividades productivas o mantener un empleo dignamente, factores que son fundamentales para que el individuo tenga un sentido de satisfacción personal $(13,14)$.

En el estudio titulado "Caracterización de las readmisiones precoces en la hospitalización psiquiátrica" que corresponde al servicio de psiquiatría de la Clínica Universidad Católica de San Carlos de Apoquindo ubicada en la zona metropolitana Este de Santiago de Chile donde se estudió y analizó una base de datos de 9 años en la cual tuvo 3.129 admisiones que corresponden a 2.610 pacientes; derivó en que: $86,13 \%$ tuvo una sola internación y $13,87 \%$ tuvieron 2 o más hospitalizaciones. De estos pacientes readmitidos el $59,4 \%$ con readmisión tardía y $40,6 \%$ con readmisión temprana. Dentro de los indicadores demográficos el resultado destacó que no se encontraron diferencias entre edad y género, siendo el promedio de edad entre 34,04 y 35,40 entre las diferentes características demográficas por tipos de hospitalización. En relación a los diagnósticos de dicha investigación se observaron más frecuentes los F30-39 (Trastornos del Humor Afectivos), los F1019 (Trastornos Mentales y del Comportamiento debidos al Consumo de Sustancias Psicotrópicas) y F20-29 (Trastornos Delirantes) (15).

La importancia de describir y categorizar la población de usuarios del servicio de internación psiquiátrica del H.C.FF.AA. podría ser utilizado para moni- torizar la realidad del servicio en sí mismo y para comparar con tendencias regionales a través de variables establecidas internacionalmente por la OMS.

\section{OBJETIVOS}

Encontrar similitudes y/o diferencias entre las características demográficas y perfiles clínicoshospitalarios de usuarios internados en el servicio de Salud Mental del H.C.FF. AA. en el período diciembre 2017 a noviembre de 2018 con un servicio similar de Chile.

Describir características demográficas (edad y sexo); conocer los diagnósticos de ingreso, la frecuencia de readmisión (temprana y tardía) y promediar los días de internación. Comparar objetivamente indicadores encontrados en la bibliografía consultada con la realidad a nivel institucional.

\section{MATERIALES Y MÉTODOS}

El procedimiento metodológico es de tipo descriptivo. Fuente de datos: la recolección de información es a través de un registro manuscrito del servicio de internación psiquiátrica del H.C.FF.AA. a partir del momento del ingreso del usuario al servicio y completada al egreso del mismo, detalla: nombre y apellido, diagnóstico de ingreso, edad, sexo, fecha de ingreso, motivo de ingreso (diagnóstico de emergencia) y fecha de egreso. Para el proceso de la información se utilizó sistema informático Microsoft Excel. Se representan los datos, con frecuencia absoluta y relativa. Se realiza la comparación de datos internacionales obtenidos de la búsqueda de información en base de datos en ciencias de la salud. Consideración ética: se cumple con los requerimientos de protección de confidencialidad de los usuarios resguardando la identidad.

\section{RESULTADOS}

En el periodo de estudio hubo un total de 147 usuarios internados que corresponden a 192 admisiones (figura 1). 93 pacientes (63\%) al sexo femenino y $54(37 \%)$ al masculino. El promedio de 
edad en años se encuentra en 41,10 (figura 2). Los diagnósticos de ingresos agrupados según CIE-10 se presentan en la figura 3. En el promedio de días de internación existe una diferencia de 5 días, siendo menor el promedio para los usuarios con solo una admisión (8,23 días) y mayor para los readmitidos más de una vez (13 días), no existiendo diferencia entre readmisiones tempranas y tardías (figura 4).

\begin{tabular}{|c|c|c|c|c|c|}
\hline $\begin{array}{c}\mathbf{N}^{\circ} \text { de } \\
\text { ingresos }\end{array}$ & Usuarios & Readmisión & $\begin{array}{c}\text { Único } \\
\text { ingreso }\end{array}$ & $\begin{array}{c}\text { Readmisión } \\
\text { temprana }\end{array}$ & $\begin{array}{c}\text { Readmisión } \\
\text { tardia }\end{array}$ \\
\hline \multirow{4}{*}{192} & F.A. & F.A. & F.A. & F.A. & F.A. \\
\cline { 2 - 6 } & 147 & 45 & 102 & 20 & 25 \\
\cline { 2 - 6 } & F.R. & F.R. & F.R. & F.R. & F.R. \\
\cline { 2 - 6 } & $(100 \%)$ & $(30,6 \%)$ & $(69,4 \%)$ & $(44,4 \%)$ & $(55,6 \%)$ \\
\hline
\end{tabular}

Figura 1. Tipo de hospitalización: Admisión y Re-Admisión.

\begin{tabular}{|c|c|c|c|c|}
\hline \multirow[b]{2}{*}{ Edades } & Adulto joven & Adulto medio & Adulto mayor & \multirow{2}{*}{$\begin{array}{c}\text { Total } \\
\text { genera }\end{array}$} \\
\hline & $18-39$ años & 40-64 años & 65 años & \\
\hline Total & 72 & 64 & 11 & 147 \\
\hline
\end{tabular}

Figura 2. Caracterización demográfica por edades.

\section{DISCUSIÓN}

El perfil de los usuarios y caracterización surgió de una base de datos con un total de 192 ingresos en el sector de internación psiquiátrica. $\mathrm{Si}$ bien no se ajusta al mismo período de tiempo estudiado en la investigación citada, se concedió la validez de las comparaciones con la realidad encontrada tanto en la cantidad de pacientes como en la prolongación del período tomado en nuestro servicio. La confiabilidad es legítima por- que los usuarios fueron ingresados al servicio por profesionales especialistas en psiquiatría desde el departamento de emergencia y tratados médicamente en el servicio por pares de la misma especialidad, los datos recabados en el servicio a su vez son extraídos de la hoja de admisión informática del Departamento de Estadística y Registros Médicos. Las tasas de demanda anual de internación psiquiátrica tuvieron una diferencia aproximada de un 50\% con inferioridad (192 usuarios) para nuestro servicio, con 347,6 usuarios para el servicio comparado. En cuanto al perfil demográfico se constató una población de $50 \%$ adultos jóvenes y una diferencia mayor en el promedio de edad de 5,22 años en comparación a la clínica relevada; en cambio el sexo femenino es considerablemente mayor y similar en ambos estudios. En relación con los diagnósticos de ingresos agrupados por CIE-10 y de mayor a menor frecuencia fueron predominantes los Trastornos Delirantes, Trastornos del Humor Afectivo seguido por los Trastornos de Personalidad con Trastornos del Comportamiento (I.A.E., Gestos Suicidas, Ideas de Muerte) y los Ingresos por Trastornos del Comportamiento debido al Consumo de Sustancias Psicotrópicas. Los demás motivos de ingresos no llegaron al 7\% del total lo que llevó a la concordancia con el resultado del estudio de aquel país ya que no existe en ese estudio motivos de ingresos como por ejemplo el diagnóstico de Retraso Mental, y el Delirium y Demencia (F00-09) es menor al $1 \%$ del total.

\begin{tabular}{|c|c|c|c|c|c|c|c|c|}
\hline \multirow{2}{*}{ Grupos diagnósticos } & \multicolumn{2}{|c|}{$\begin{array}{l}\text { Sin } \\
\text { readmisión }\end{array}$} & \multicolumn{2}{|c|}{$\begin{array}{c}\text { Readmisión } \\
\text { temprana }\end{array}$} & \multicolumn{2}{|c|}{$\begin{array}{c}\text { Readmisión } \\
\text { tardía }\end{array}$} & \multicolumn{2}{|c|}{$\begin{array}{c}\text { Total por } \\
\text { diagnóstico }\end{array}$} \\
\hline & F.A. & F.R. & F.A. & F.R. & F.A. & F.R. & F.A. & F.R. \\
\hline F00-09 Trastornos mentales orgánicos & 3 & 2,94 & & & & & 3 & 2 \\
\hline F10-19 Trastornos mentales y del comportamiento por consumo de sustancias & 22 & 21,56 & 2 & 10 & & & 24 & 16,3 \\
\hline F20-29 Esquizofrenia y trastornos de ideas delirantes & 21 & 20,58 & 5 & 25 & 14 & 56 & 40 & 27,2 \\
\hline F30-39 Trastornos del humor (afectivos) & 24 & 23,52 & 8 & 40 & 6 & 24 & 38 & 25,8 \\
\hline F40-49 Trastorno neurótico & 2 & 1,96 & & & & & 2 & 1,4 \\
\hline F60-69 Trastorno de personalidad con trastorno del comportamiento & 27 & 26,47 & 3 & 15 & 5 & 20 & 35 & 23,8 \\
\hline F70-79 Retraso mental & 3 & 2,94 & 2 & 10 & & & 5 & 3,4 \\
\hline Total & 102 & 100 & 20 & 100 & 25 & 100 & 147 & 100 \\
\hline
\end{tabular}

Figura 3. Porcentaje de distribución por grupos diagnósticos y tipo de readmisión. 
Respecto al tipo de hospitalización hubo una concordancia que la gran mayoría en el tiempo estudiado tuvo solo una admisión hospitalaria, se observó la similitud en readmisiones en ambos estudios siendo menor a la mitad readmisiones tempranas, y mayor a la mitad readmisiones tardías del total de pacientes con readmisiones.

\begin{tabular}{|c|c|c|c|c|c|}
\hline \multicolumn{2}{|c|}{ Sin readmisión } & \multicolumn{2}{c|}{ Readmisión temprana } & \multicolumn{2}{c|}{ Readmisión tardía } \\
\hline F.A. & Media & F.A. & Media & F.A. & Media \\
\hline 102 & $8,23 \mathrm{~d}$ & 20 & $13,66 \mathrm{~d}$ & 25 & $13,628 \mathrm{~d}$ \\
\hline
\end{tabular}

Figura 4. Duración de estadía hospitalaria según tipo de hospitalización.

Existe la similitud en el tipo de diagnóstico en pacientes con readmisión temprana siendo mayor los Trastornos del Humor Afectivos seguido de los Trastornos con Ideas Delirantes los más representativos. Estos 2 grupos de diagnósticos también representan la mayoría de aquellos readmitidos tardíamente pero a la inversa y se suma tercero en frecuencia con un quinto del total de readmitidos tardíamente el grupo de diagnóstico Trastorno de Personalidad con Trastornos del Comportamiento. El resultado es que estos grupos de diagnósticos varían entre ellos significativamente dentro de los grupos de readmisiones como también sucede en la clínica comparada a nivel regional.

\section{CONCLUSIÓN}

Se realizó un estudio retrospectivo y descriptivo con caracterización de perfil clínico-demográfico de 147 pacientes que ingresaron y fueron tratados médicamente en el servicio de internación psiquiátrica de la División Salud Mental del H.C.FF.AA. de la República Oriental del Uruguay durante un año.

La tasa anual baja de internación psiquiátrica revela que una gran cantidad de individuos se re-insertan de forma adecuada a la comunidad, indicando que la atención ambulatoria post internación tiene una buena calidad y con ello el sentido de satisfacción personal de estos pacientes. Se estableció que la diferencia de sexo está liga- da a los porcentajes de la población general y el sexo femenino ingresado y tratado representa a la extensión del derecho generado por el usuario activo y retirado titular.

Los diagnósticos dominantes fueron aquellos en los cuales el tipo de enfermedad tiene características de cronicidad y recidiva propias, como son los grupos de los Trastornos Psicóticos y los Trastornos del Humor Afectivos en sus entidades patológicas Esquizofrenias y Depresión Mayor. Entidades patológicas también relacionadas con la readmisión precoz y tardía. La predominancia del sexo femenino en los reingresos se sostuvo vinculada al número total de usuarios ingresados. Se destaca la importancia de seguir realizando en nuestro quehacer cotidiano profesional la tarea de investigación para poder seguir tendencias a nivel nacional y regional e ir en el trazado de las políticas continentales como la "unidad técnica de la OPS", dentro del Departamento de Enfermedades no Transmisibles y Salud Mental, promoviendo, coordinando e implementando actividades de cooperación técnica dirigidas a fortalecer las capacidades nacionales para desarrollar políticas, planes, programas y servicios, contribuyendo a promover la salud mental, reduciendo la carga que significan las enfermedades psíquicas, previniendo las discapacidades y desarrollando la rehabilitación.

DECLARACIÓN DE CONFLICTOS DE INTERESES: Los autores no reportan ningún conflicto de interés. El estudio se realizó con recursos propios de los autores y/o la institución a la que representan. 


\section{REFERENCIAS}

(1) Ramos A, Vázquez JL, Herrera S. CIE-10 (I): Introducción, historia y estructura general.

Papeles Méd 2002; 11(1):24-35.

(2) World Health Organization. Guía de bolsillo de la clasificación CIE-10: clasificación de los trastornos mentales y del comportamiento. Madrid : Editorial Médica Panamericana, 2000. p.1-16.

(3) Organización Mundial de la Salud. Salud Mental un estado de bienestar. [Internet]. [Citado 2019 Mar 09]. 1 pantalla. Disponible en: https://www.who. int/features/factfiles/mental_health/es/

(4) Herrero Jaén S. Formalización del concepto de salud a través de la lógica: impacto del lenguaje formal en las ciencias de la salud. Ene. [Internet]. 2016 Ago [citado 2019 Abr 29]; 10(2). Disponible en: http://scielo.isciii.es/scielo.php? script=sci_arttext\&pid=S1988-348X2016000200 006\&lng $=e s$

(5) González M, Carreño M. Psiquiatría De Enlace y Medicina De Enlace, Nuevos Alcances.

Revista Médica Clínica Las Condes 2017; 28(6):944-8. doi: 10.1016/j.rmclc.2017.10.012

(6) Sperry L, Sperry J. Cognitive Behavior Therapy of DSM 5 Personality Disorders: Assessment, Case Conceptualization, and Treatment. $3^{\text {rd }}$. edition. New York : Routledge, Taylor \& Francis, 2015. 244 p. https://doi.org/10.4324/9780203764084

(7) Sperry L. Handbook of Diagnosis and Treatment of DSM-5 Personality Disorders Assessment, Case Conceptualization, and Treatment, Third Edition. New York : Routledge, 2016. 316 p. https://doi.org/10.4324/9780203763728
(8) Lurigio AJ. DSM-5. Salem Press Encyclopedia of Health [Internet]. 2018 [Citado 2019 Abr 09]; Disponible en: http://search.ebscohost.com.proxy. timbo.org.uy:2048/login.aspx?direct=true $\& d b=$ ers\&AN=115297534\&lang=es\&site=eds-live

(9) American Psychiatric Association. Diagnostic and Statistical Manual of Mental Disorders. 5th. Edition (DSM-5). Washington, DC, 2013.

(10) Gutiérrez I, Montero A, Rodríguez E, Fernández A. Enfermedad psiquiátrica. Medicine 2015; 11(85):5055-63. https://doi.org/10.1016/j.med. 2015.08.001

(11) Pichot P, coord. DSM-IV: Manual diagnóstico y estadístico de los trastornos mentales.

Barcelona : Masson, 1995. p.401-456.

(12) Organización Panamericana de la Salud. Organización Mundial de la Salud. Salud Mental. [Internet]. [Citado 2019 Set 03]. 1 pantalla.

Disponible en: https://www.paho.org/hq/index.php? option=com_topics $\&$ view $=$ article $\& i d=210 \&$ Item$\mathrm{id}=40242$ \&lang $=\mathrm{es}$

(13) Díaz Soto CM, Orozco Moreno AJ, Villán Ramírez NC. Factores asociados con la readmisión de pacientes psiquiátricos en el oriente antioqueño en 2014. Medicina UPB 2016; 35(1):17. https://dx.doi.org/10.18566/medupb.v35n1.a03

(14) Sánchez R, Jaramillo LE, Herazo MI. Factores asociados a rehospitalización temprana en psiquiatría. Biomédica 2013; 33:276-282.

(15) Weiss MC, Santander TJ, Aedo Cl, Fuentes MX. Caracterización de las readmisiones precoces en la hospitalización psiquiátrica. Revista chilena de neuro-psiquiatría [Internet]. 2013 [citado 2019 Mar 9]; 51(4):239-244. http://dx.doi.org/10.4067/S0717-92272013000400002 